\title{
Synchronous adenocarcinoma and primary malignant lymphoma of the stomach
}

\author{
A.B. Akosa' ${ }^{1}$ D.M. Clark ${ }^{1}$ and L. Desa ${ }^{2}$ \\ Departments of ${ }^{1}$ Histopathology and ${ }^{2}$ General Surgery, Royal Postgraduate Medical School, \\ Hammersmith Hospital, Ducane Road, London W12 0HS, UK.
}

Summary: A synchronous presentation of an adenocarcinoma and a primary low grade B cell lymphoma of the stomach is reported in a 79 year old man. Both diagnoses were made on endoscopic biopsies and immunocytochemistry was essential in confirming the lymphoma. The true incidence, aetiology and spatial relations of these tumours are unknown since only few cases of such synchronous gastric tumours have been reported in the literature.

\section{Introduction}

Ninety-five per cent of gastric malignancies are adenocarcinomas of which $1-2 \%$ are multiple. Primary gastric lymphomas make up $60 \%$ of the non-epithelial malignancies. The synchronous presentation of both tumours, however, is uncommon. In 2438 consecutive cases of resected gastric carcinoma in a 14-year period (1974-1988), the association of primary gastric lymphoma and carcinoma was found in only two cases $(0.08 \%){ }^{1}$ Few cases of these synchronous tumours have been reported outside Japan. ${ }^{2-6}$

We present one such case of synchronous gastric adenocarcinoma and lymphoma.

\section{Case report}

A 79 year old male presented with a 3-week history of progressive dysphagia, initially for solids and later for semi-solids. He had been a heavy smoker in the past, lost two stones in weight and appeared cachectic. There was no lymphadenopathy, pallor or icterus and systemic examination was unremarkable. In particular, hepatosplenomegaly was absent. The chest X-ray was normal. However, a barium swallow showed an extensive $8 \mathrm{~cm}$ stricture of the lower end of the oesophagus which was confirmed on endoscopy. In addition, an ulcerated lesion $4 \mathrm{~cm}$ in diameter was seen on the lesser curve of the stomach. Cytological brushings were taken from the stricture and the biopsies were from the lower oesophagus, cardia, lesser curvature and duodenum. Blood profile was normal and a bone marrow aspirate performed after the diagnosis of

Correspondence: A.B. Akosa, D.C.P., M.R.C.Path. Accepted: 3 April 1990 gastric lymphoma was also normal. Abdominal ultrasound revealed no intra-abdominal masses.

Surgical treatment, which would have entailed an oesophagogastrectomy was considered hazardous in view of his age and poor general condition. Palliative treatment with nutritious supplements and opiates was therefore instituted.

Multiple endoscopic biopsies from the lower end of the oesophagus (36,37 and $38 \mathrm{~cm})$, cardia, lesser curve and duodenum in $10 \%$ formal saline were conventionally processed and stained with haematoxylin and eosin. Cytological smears were stained by Papanicolaou and Giemsa stains. Biopsies from the lesser curve were also stained using the unlabelled peroxidase antiperoxidase (PAP) method to leucocyte common antigen (LCA), L26, UCHL1, Cam 5.2, kappa and lambda immunoglobulin light chains (Table I).

Malignant cells from an adenocarcinoma were seen on the brushings. A moderately differentiated adenocarcinoma of the cardia (Figure 1) extending to the lower end of the oesophagus was identified. The biopsies from the ulcer on the lesser curve showed a predominantly dense monomorphic infiltrate of centrocyte-like cells displacing crypts and infiltrating into the submucosa with the occasional lymphoid follicle; lymphoepithelial lesions were present but sparse (Figure 2). Plasma cells were present beneath the surface epithelium. Initial assessment called into question a florid lymphoid reaction to the carcinoma or a lymphoma. All the cells were LCA and L26 positive and Cam 5.2 and UCHL1 negative. Light chain restriction for kappa indicative of monoclonality was demonstrated confirming a low grade B cell lymphoma. Duodenal biopsies revealed a mild duodenitis. 
Table I Immunoreagents, source and reactivity

\begin{tabular}{lll}
\hline Immunoreagents & Source & \multicolumn{1}{c}{ Reactivity } \\
\hline LCA & Dako & All haemopoietic cells \\
L26 & Dako & B lymphocytes \\
UCHLI & Dako & T lymphocytes \\
Cam 5.2 (Cytokeratin) & Dako & Malignant epithelial cells \\
Kappa \& Lambda & Dako & Immunoglobulin light chains \\
\hline
\end{tabular}

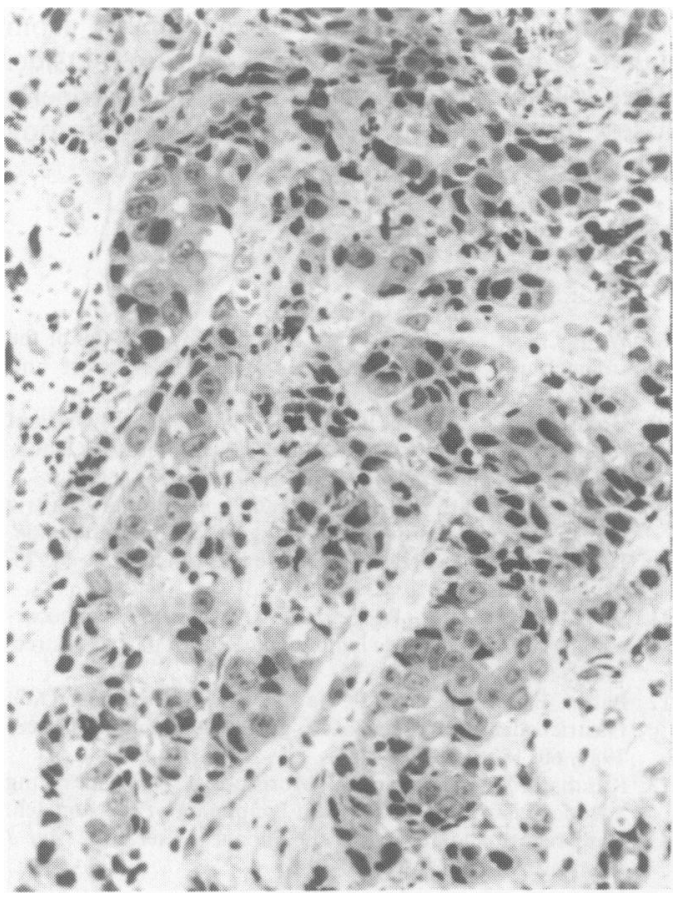

Figure 1 Malignant well and poorly formed glands and small clusters of malignant cells in the lamina propria with lymphocytic infiltrate. $H \& E \times 128$.

\section{Discussion}

The 'field of growth' theory of tumourigenesis explains tumour multi-focality and multiple tumours of identical type. The simultaneous presence of two different tumours (epithelial and non-epithelial) in the same organ presents a puzzle not least in terms of aetiology. It has been suggested that the tumours are caused independently by the same aetiological agent(s). Alternatively, either the carcinoma develops as a result of chronic irritation of the mucosa by the lymphoma or the lymphoma arises as a consequence of the host tissue response to the gastric adenocarcinoma. ${ }^{5}$ It is practically impossible to determine which of the tumours antedates the other. a
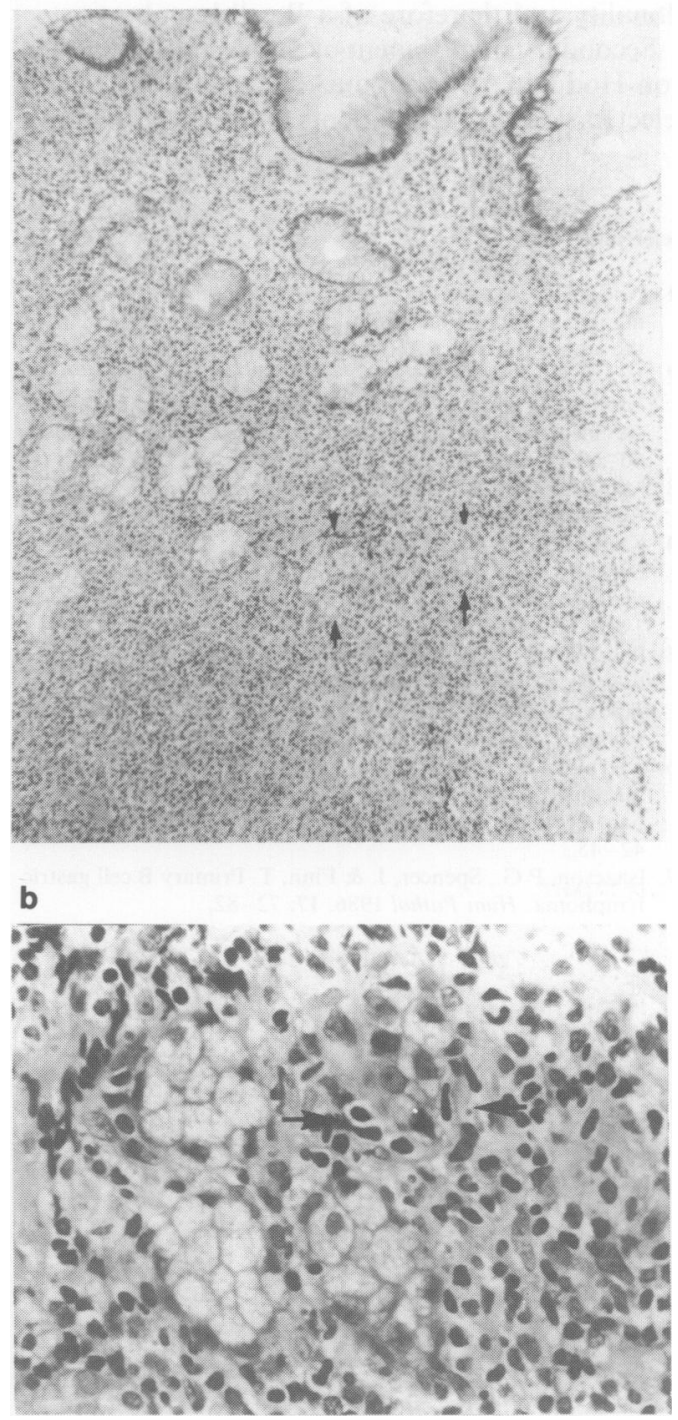

Figure 2 (a) A dense lymphocytic infiltrate in the lamina propria with spacing out of crypts: some of the latter appear to be destroyed (arrows). H\&E $\times 41$. (b) High power showing infiltration of glands by elongated centrocyte-like cells (arrows), constituting a lymphoepithelial lesion. H\&E $\times 165$. 
A florid host tissue response consisting of a dense infiltrate of lymphoid cells can mimic lymphomas histologically, more so in lymphomas of gut associated lymphoid tissue in which cellular polymorphism is a feature. ${ }^{7}$ The relationship between a florid lymphoid infiltrate and lymphoma in the stomach is unclear; however, gastric lymphomas preceded by focal lymphoid hyperplasia have been reported. ${ }^{8}$ Immunocytochemistry is essential to distinguish between lymphomas and reactive lymphoid infiltrates particularly those of $B$ cell lineage. Light chain restriction is diagnostic of monoclonality and therefore of a B cell lymphoma.

Secondary involvement of the stomach occurs in non-Hodgkin's lymphoma. In a consecutive unselected study of $\mathbf{4 2 3}$ patients with non-Hodgkin's lymphoma, $3 \%$ (13 patients) showed evidence of gastric involvement. ${ }^{9}$ All these patients, however, had systemic disease, including lymphadenopathy. Our patient showed no evidence of systemic disease.

Gastric adenocarcinoma has been reported in patients treated for prior lymphomas of the stomach. ${ }^{10-12}$ In these cases, partial gastrectomy by itself, as well as radiotherapy and chemotherapy have been implicated as predisposing factors. ${ }^{4}$

Thirty-six cases of synchronous adenocarcinoma and lymphomas have been reported from Japan, ${ }^{1,13}$ in contrast to the sporadic cases outside. These numbers are, however, too small to properly assess the true incidence and the factors affecting the prognosis of these tumours.

\section{References}

1. Noda, T., Akashi, H., Matsueda, S. et al. Collison of malignant lymphoma and multiple early adenocarcinoma of the stomach. Arch Pathol Lab Med 1989, 113: 419-422.

2. Jernstrom, P. \& Murray, G.C. Synchronous double primary lymphosarcoma and adenosarcoma (Collision tumour) of the stomach with cancer to cancer metastasis. Cancer 1966, 19: 60-66.

3. Manier, J.W. \& Reyes, C.N. Collision tumour of the stomach. Gastroenterology 1974, 67: 1011-1015.

4. Kane, E.P., Weingarten, L.A., Payson, B.A., Mori, K. \& Sarlin, J.G. Synchronous ulcerating adenocarcinoma and malignant lymphoma of the stomach. Am J Gastroenterol 1982, 77: $461-463$.

5. Planker, M., Fischer, J.Th., Peter, U. \& Borchard, F. Synchronous double primary malignant lymphoma of low grade malignancy and early cancer (collision tumour) of the stomach. Hepatogastroenterology 1984, 31: 144-148.

6. Czerniak, A., Cotan, G., Engelberg, I.S. et al. The simultaneous coexistence of adenocarcinoma and primary malignant lymphoma in the stomach. $J$ Surg Oncol 1985, 30: 42-45.

7. Isaacson, P.G., Spencer, J. \& Finn, T. Primary B cell gastric lymphoma. Hum Pathol 1986, 17: 72-82.

8. Wolf, J.A. \& Spjut, H.J. Focal lymphoid hyperplasia of the stomach preceding gastric lymphoma. Case report and review of the literature. Cancer 1981, 48: 2518-2523.

9. Goffinet, D.R., Warnke, R., Dunnick, N.R. et al. Clinical and surgical (laparotomy) evaluation of patients with non Hodgkin's lymphoma. Can Treat Rep 1977, 61: 981-992.

10. Shani, A., Schutt, A.J. \& Weiland, L.H. Primary gastric malignant lymphomas followed by gastric adenocarcinoma. Reports of 4 cases and review of the literature. Cancer 1978, 42: 2039-2044.

11. Sellin, J., Levin, B., Reckerd, C. et al. Gastric adenocarcinoma following gastric lymphoma. Role of partial gastrectomy. Cancer 1980, 45: 996-1000.

12. Baron, B.W., Bitter, M.A., Baron, J.M. \& Bostwick, D.G. Gastric adenocarcinoma after gastric lymphoma. Cancer 1987, 60: 1876-1882.

13. Kasahara, Y., Takemoto, M., Morishita, A. et al. Coexisting adenocarcinoma and malignant lymphoma of the stomach. Case report and review of the Japanese literature. Am J Gastroenterol 1988, 83: 190-193. 